\title{
ON ENERGETICS AND STRUCTURE OF SUB-PARSEC SCALE JETS IN QUASARS
}

\author{
M. SIKORA \\ N. Copernicus Astronomical Center, Polish Academy of Sciences, \\ Bartycka 18, 00-716 Warsaw, Poland \\ E-mail: sikora@camk.edu.pl \\ G. MADEJSKI \\ Stanford Linear Accelerator Center, \\ Menlo Park, CA 94025, USA \\ E-mail: madejski@slac.stanford.edu
}

\begin{abstract}
In our review of sub-parsec scale jets in quasars, we discuss the following issues: observations of parsec and sub-parsec scale jets; energy dissipation and particle acceleration; radiative processes; magnetic fields, pair content and energetics; variability and its relation to the central engine activity. In particular, we describe how internal shocks can explain properties of gamma-ray flares and demonstrate that $\mathrm{MeV}$ blazars (those with luminosity peak in the $1-30 \mathrm{MeV}$ range) can be unified with $\mathrm{GeV}$ blazars (those with luminosity peak at $\mathrm{GeV}$ energies) assuming that in $\mathrm{GeV}$ blazars the gamma-ray flares are produced via Comptonization of broad emission lines, whereas in the $\mathrm{MeV}$ blazars they result from Comptonization of infrared radiation of hot dust. We also make predictions about the radiative effects of bulk Compton process in the soft X-ray band and show how spectral and variability properties in that band can be used to constrain structure of jets near their bases.
\end{abstract}

\section{Introduction}

Observational data imply that jets in radio-loud quasars can extend over 8 decades of distance, from milliparsecs to hundreds of kiloparsecs. Such jets are launched in the vicinity of super-massive black holes and transport energy at a rate which is sometimes comparable with the accretion luminosity, i.e. $10^{45}-10^{47} \mathrm{erg} \mathrm{s}^{-1}$. 24 4 . Jets are relativistic and therefore Doppler boosting often makes them observable only on one side of the quasar. However, the hot spots where jets terminate are usually seen on both sides of the central source, and emission on both sides shows similar flux. This suggests that the radiation from hot spots is nearly isotropic, and indicates that jets are "light", i.e. have lower mass densities than the external medium. The lightness is necessary to explain the nonfelativistic speeds of hot spots and formation of extended radio lobe structures. 1 Using the asymmetry of radio brightness of jets and counterjets, Wardle and Aaron 36 derived the bulk Lorentafactor $\Gamma \sim 3$. Larger Lorentz factors are suggested by $\mathrm{X}$-ray observations 3.33 ; however, because $\mathrm{X}$ - 
ray sensitivity is too low to observe counter-jets, such estimates are not direct and depend on the details of models of the $\mathrm{X}$-ray production.

Quasar jets are imaged in the radio band down to parsec scales. There, they join the central cores, which in turn, due to the synchrotron self-absorption, produce very flat spectra, with the energy spectral index $\alpha<0.5\left(\alpha: F_{\nu} \propto\right.$ $\nu^{-\alpha}$ ) (see, e.g., review by Zensus. 37 In quasars with jets oriented close to the line of sight, the flux of the radio cores strongly dominates over that from radio lobes, and the total radio spectra are flat. Because of this, core-dominated radio quasars are often called FSRQs (flat spectrum radio quasars), as opposed to the lobe dominated radio quasars, which have steep radio spectra and are called SSRQs (steep spectrum radio quasars). Radio cores in FSRQs are often variable on monthly time scales, and variability of the radio flux is often accompanied by an appearance of ejecta propagating with apparently superluminal velocities. 34.16

The physics of the parsec scale jets (energetics, magnetic fields, particle distribution) is determined by the use of images, spectra, variability, and polarization properties. In particular, the angle of linear polarization provides information about magnetic field orientation relative to the jet axis. Recent data suggest that such orientation changes from being perpendicular to the jet closer to the central source to the roughly parallel at somewhat larger distances. 32,19 This may indicate that at smaller distances, the jets are free (ballistic) and that their energy is dissipated mainly in perpendicular shocks resulting from collisions of the jet inhomogeneities moving with different radial velocities. At larger distances, on the other hand, most dissipation is likely to come from oblique, reconfinment shocks produced due to interaction of a jet with external medium. For a number of objects, there are also circular polarization measurements available. 12 If circular polarization is produced via the Faraday conversion mechanism, it provides information on the number of electrons at mildly relativistic energies. Combined with the information about the total power of a jet, this can be used to estimate the pair content. It should be emphasized that no information about number of mildly relativistic electrons is available from direct radio flux observations, because the synchrotron radiation by mildly relativistic electrons is self-absorbed.

The parts of jets located close to the cores can be studied also in other spectral bands besides radio, despite the fact that they are by many orders too compact to be resolved. In most FSRQs, typical thermal components of the quasar emission - such as the UV radiation from an accretion disc, the $\mathrm{X}$-ray radiation from the disc corona, and the infrared radiation from dust are overshined by the Doppler-boosted nonthermal jet radiation. Variability of this radiation is measured on time scales often shorter than those observed in 
the radio band, and this strongly suggests its parsec/sub-parsec origin. High polarization in the optical and IR bands implies synchrotron mechanism, while the distinct high energy components, with luminosity peaks in the $\mathrm{MeV}-\mathrm{GeV}$ range, 5 are presumably products of Comptonization of broad emission lines and IR radiation of hot dust. 28 If If the EC (external-Compton) scenario is indeed responsible for the high energy components, then observations of the $\mathrm{X}$-ray spectra can provide an exceptional opportunity to study the population of the lower energy population of the relativistic electrons directly. 29 Furthermore, due to narrowness of the electromagnetic spectrum bands covered by dominant broad emission lines and by infrared radiation of hot dust, any features such as breaks in the energy distribution of electrons should be sharply imprinted in the EC radiation components. In particular, as it will be demonstrated below, the shapes of the observed high energy spectra are consistent with the two-power-law injection function of electrons, and the latter can result from a two-step acceleration process of electrons as discussed below.

The two component nonthermal spectra are also observed in BL Lac objects, which differ from FSRQs as having very weak or undetectable emission lines. FSRQs and BL Lac objects form together a class of objects called blazars. It is shown by Fossati et al. 11 that broadband spectra of blazars form a sequence which can be parametrized by their total luminosities. In this sequence, FSRQs are the most luminous objects. Both their low and high energy spectral components appear to be least extended to the high energies, and their $\gamma$-ray luminosities during flares strongly dominate over synchrotron luminosities. The least luminous blazars are represented by the X-ray selected BL Lac objects. Their synchrotron spectra extend up to hard X-rays, and the $\gamma$-ray spectra reach $\mathrm{TeV}$ energies. $\gamma$-ray luminosities in the TeV-emitting BL Lac objects usually do not dominate over synchrotron luminosities. Low luminosity BL Lac objects are probably associated with nuclei of radio galaxies accreting at a low accretion rate, and $\gamma$-rays are very likely produced by the SSC (synchrotron-self-Compton) process. In the more luminous, radio selected BL Lac objects the EC process can be dominant, 20 in similarity to FSRQs.

In this presentation we focus on jets in quasars, where the interaction of the jet with external radiation is significant and the presence or lack of radiative effects of this interaction can be used to determine physical parameters and constraints on structure of jets on sub-parsec scales. This paper is organized as follows: in $\S 2$, we analyze the energetics of intrinsic collisions of material in the jet; in $\S 3$, we present the motivation for introduction of the two-step stochastic acceleration of electrons; in $\S 4$, we discuss the formation of spectral breaks in the high energy spectral components; in $\S 5, \S 6$, and $\S 7$, respectively, we derive the magnetic field intensity, the pair content, and the average power of a jet. 
In $\S 8$, we formulate our predictions regarding the production of soft $\mathrm{X}$-ray precursors via Comptonization of the external radiation by the cold electrons in a jet, and list the main conclusions in $\S 9$.

\section{Energy dissipation}

Cooling time scales of ultrarelativistic electrons, which in quasar jets produce synchrotron radiation in the optical band and inverse-Compton radiation in the $\mathrm{MeV}-\mathrm{GeV}$ range, is much shorter than the dynamical/propagation time scale. Therefore, such electrons must be accelerated in situ, in sites where the jet loses part of its energy and the shocks are formed. The jet energy can be dissipated in internal shocks - formed in collisions betyeen inhomogeneities in a jet 2831 or following reconnection of magnetic fields, 25 - and for in external shocks, formed via reconfinment of the jet by external medium 26, 17 or due to collisions of a jet with external clouds 8 . Perpendicular orientation of magnetic fields to the jet axis, inferred from the polarization measurements in the optical band 13 and at the high radio frequencies 19 favors the scenario where at subparsec distances, the particles are accelerated in transverse shocks which are likely to be produced via collisions of inhomogeneities flowing down the jet with different radial velocities. A given collision event is then responsible for a given flare, and the time scale of such a collision, as measured in the comoving frame of the shocked plasma, is

$$
t_{\text {coll }}^{\prime} \simeq t_{f l} \mathcal{D},
$$

where $t_{f l}$ is the observed time scale of the flare, and

$$
\mathcal{D} \equiv \frac{1}{\Gamma\left(1-\beta \cos \theta_{\text {obs }}\right)}
$$

is the Doppler factor of the shocked plasma. The amount of energy dissipated during the collision is

$$
E_{\text {diss }}^{\prime} \simeq \frac{L_{f l}^{\prime} t_{\text {coll }}^{\prime}}{\eta_{\text {rad }} \eta_{e}} \simeq \frac{L_{f l} t_{f l}}{f \mathcal{D}^{3} \eta_{\text {rad }} \eta_{e}},
$$

where $\eta_{e}$ is the fraction of dissipated energy used to accelerate electrons, $\eta_{\text {rad }}$ is the (average) radiative efficiency of electrons, and

$$
L_{f l} \equiv 4 \pi\left(\frac{\partial L_{f l}}{\partial \Omega}\right)=4 \pi\left(\frac{\partial L_{f l}^{\prime}}{\partial \Omega^{\prime}}\right) \mathcal{D}^{4}=L_{f l}^{\prime} f \mathcal{D}^{4}
$$


is the apparent luminosity. All primed quantities are as measured in the source comoving frame. The factor $f$ expresses the additional dependence of the apparent luminosity on the observed angle, if the intrinsic radiation is anisotropic. For the EC process, $f \simeq(\mathcal{D} / \Gamma)^{2}$, while for synchrotron and SSC radiation $f \simeq 1$.

In the above relations it was assumed that during a collision, the shocked plasma moves with a constant bulk Lorentz factor. In general this is not true: depending on the initial parameters of inhomogeneities, the shock can accelerate or decelerate, and during the collision the shock structure can evolve from a double one to a single shock (forward or reverse). However, if inhomogeneities are intrinsically identical, then the forward-reverse shock structure is symmetric in the shocked plasma frame (= discontinuity contact surface frame), and the bulk Lorentz factor of the shock is constant. This case is adopted in our quantitative analysis, not only for simplicity, but also because it provides the highest efficiency of energy dissipation.

Assuming that the inertia of inhomogeneities is dominated by protons (i.e. $\left.n_{e} / n_{p} \ll m_{p} / m_{e}\right)$, and noting that

$$
E_{\text {diss }} \equiv \eta_{\text {diss }}\left(E_{1}+E_{2}\right)=\Gamma E_{\text {diss }}^{\prime},
$$

where $E_{1} \simeq\left(N_{p} / 2\right) \Gamma_{1} m_{p} c^{2}$ and $E_{2} \simeq\left(N_{p} / 2\right) \Gamma_{2} m_{p} c^{2}$ are energies of inhomogeneities, we find that total number of protons involved in the collision is

$$
N_{p} \simeq \frac{2 \Gamma E_{\text {diss }}^{\prime}}{\eta_{\text {diss }}\left(\Gamma_{1}+\Gamma_{2}\right) m_{p} c^{2}} \simeq \frac{2 L_{f l} t_{f l}}{\kappa f \mathcal{D}^{3} \eta_{\text {rad }} \eta_{e} m_{p} c^{2}},
$$

where

$$
\kappa \equiv \frac{E_{d i s s}^{\prime}}{N_{p} m_{p} c^{2}}=\frac{\eta_{d i s s}\left(\Gamma_{1}+\Gamma_{2}\right)}{2 \Gamma}
$$

is amount of energy dissipated per proton in units of $m_{p} c^{2}$. For $\Gamma_{2}>\Gamma_{1} \gg 1$, energy and momentum conservations give

$$
\Gamma \simeq \sqrt{\Gamma_{1} \Gamma_{2}}
$$

and

$$
\eta_{\text {diss }} \simeq \frac{\left(\left(\Gamma_{2} / \Gamma_{1}\right)^{1 / 2}-1\right)^{2}}{\left(\Gamma_{2} / \Gamma_{1}\right)+1}
$$

and then

$$
\kappa \simeq \frac{\left(\left(\Gamma_{2} / \Gamma_{1}\right)^{1 / 2}-1\right)^{2}}{2\left(\Gamma_{2} / \Gamma_{1}\right)^{1 / 2}}
$$

For $\Gamma_{2} / \Gamma_{1}=2.5$, which corresponds with $\eta_{\text {diss }} \simeq 0.1, \kappa \simeq 0.11$. 


\section{Particle acceleration}

As it was demonstrated by Sikora and Madejski, 29 energy flux in powerful jets in quasars cannot be dominated by pair plasma. This is because such jets would produce much larger fluxes of soft X-ray radiation than is observed. Hence, we assume that the inertia of jets is dominated by protons. In this case, the structure of shocks and the structure of the turbulence around them are both determined by protons. Provided that the magnetic fields are amplified up to equipartition with protons, the protons are effectively accelerated both by 1 st order and by 2nd order Fermi process. 15 Those which reach energies $>10^{9} \mathrm{GeV}$, interact efficiently with ambient photons and trigger (mainly via photo-meson process) the synchrotron-supported pair cascades. 21 However, such a model fails to reproduce very hard X-ray spectra of FSRQs. 30 This may indicate that too few protons reach sufficiently high energies to power pair cascades.

Alternative possibility is that the high energy radiation in FSRQs is produced by directly accelerated electrons. However, for the acceleration of electrons to occur via the Fermi process, these electrons must be first preheated/preaccelerated up to energies $\gamma_{b}$, at which point, the magnetic rigidity of electrons becomes comparable with magnetic rigidity of thermal protons, i.e. when their momenta are equal:

$$
m_{e} \sqrt{\gamma_{b}^{2}-1} \simeq m_{p} \sqrt{\gamma_{p, t h}^{2}-1}
$$

where

$$
\gamma_{p, t h}-1=\eta_{p, t h} \kappa
$$

is the average thermal proton energy in the shocked plasma, and $\eta_{p, t h}$ is the fraction of the dissipated energy tapped to heat the protons. For the reasonable assumption that $\Gamma_{2} / \Gamma_{1}<10, \gamma_{p, t h}-1<1$, i.e. the thermal proton plasma is at most mildly relativistic.

Noting that the average energy of injected electrons is

$$
\bar{\gamma}_{i n j}-1 \simeq \frac{n_{p} m_{p}}{n_{e} m_{e}} \eta_{e} \kappa=\frac{n_{p} m_{p}}{n_{e} m_{e}} \frac{\eta_{e}}{\eta_{p, t h}}\left(\gamma_{p, t h}-1\right),
$$

we find that for $\gamma_{b}$ and $\bar{\gamma}_{i n j} \gg 1$

$$
\frac{\gamma_{b}}{\bar{\gamma}_{i n j}} \sim \frac{n_{e}}{n_{p}} \frac{\eta_{p, t h}}{\eta_{e}}\left(\frac{\gamma_{p, t h}+1}{\gamma_{p, t h}-1}\right)^{1 / 2} .
$$

Hence, for non- or mildly relativistic shocks and for $\eta_{p, t h} \sim \eta_{e}$, the threshold energy for the Fermi acceleration of electrons significantly exceeds the average 
energy of electrons, even if $n_{e}=n_{p}$. That implies that the often-considered bulk preheating process is not able to provide an adequate number of electrons with $\gamma \geq \gamma_{b}$. But, as it was recently demonstrated in numerical PIC (particlein-cell) simulations, the preheating/preacceleration mechanism has a stochastic character and a significant fraction of electrons can reach such energies. 0.27 Furthermore, the fact that in FSRQs, the X-ray spectral index $\alpha_{X}$ is never negative, implies that most of electrons occupy the lowest energies, i.e. the median Lorentz factor is $\ll \bar{\gamma}_{i n j}$. Therefore, this scenario does not predict a thermal bump in the preaccelerated electron energy distribution. Instead, the preacceleration mechanism, in similarity to the Fermi process, produces a power-law energy distribution of electrons. It should be emphasized here that because the X-rays in FSRQs are produced in the slow cooling regime (see next Section), electrons with low energies are not populated by cooling effect, but are directly injected with such energies.

\section{Electromagnetic spectra}

The basic feature of the high energy spectra in FSRQs - a spectral break between the X-ray and the $\gamma$-ray bands - has a natural explanation in terms of the EC model. In this model, X-ray spectra are produced by electrons with radiative cooling time scale $t_{\text {cool }}^{\prime}$, longer than the collision time scale $t_{\text {coll }}^{\prime}$ (slow cooling regime), whereas $\gamma$-rays are produced by electrons with $t_{\text {cool }}^{\prime}<t_{\text {coll }}^{\prime}$ (fast cooling regime). Noting that the angle averaged cooling rate of electrons, dominated by Comptonization of external radiation, is

$$
|\dot{\gamma}| \simeq \frac{\sigma_{T}}{m_{e} c} u_{e x t}^{\prime} \gamma^{2}
$$

we obtain that the angle averaged cooling time scale is

$$
t_{\text {cool }}^{\prime} \simeq \frac{\gamma}{|\dot{\gamma}|} \simeq \frac{m_{e} c}{\sigma_{T}} \frac{1}{\gamma u_{e x t}^{\prime}},
$$

where $u_{e x t}^{\prime}$ is the energy density of an external radiation field. Then, from $t_{\text {cool }}^{\prime}=t_{\text {coll }}^{\prime}$, where $t_{\text {coll }}^{\prime}$ is given by Eq. (1), the break in the electron distribution is at energy

$$
\gamma_{c} \simeq \frac{m_{e} c}{\sigma_{T}} \frac{1}{u_{e x t}^{\prime} t_{f l} \mathcal{D}} .
$$

For $\gamma<\gamma_{c}$, the slope of the electron distribution is the same as the slope of the injection function; for $\gamma>\gamma_{c}$, the slope of the electron energy distribution is steeper by $\Delta s=1\left(s: N_{\gamma} \propto \gamma^{-s}\right)$. 
Since time scales of flares in FSRQs are rarely shorter than 1 day, the distances from the central source where they are produced,

$$
r_{f l} \sim\left(r_{f l} / \Delta r_{c o l l}\right) c t_{f l} \mathcal{D} \Gamma,
$$

are expected to be larger than 0.1 parsec. At such distances, contribution to $u_{e x t}^{\prime}$ is dominated by the diffuse components of the broad emission line light and of the infrared radiation of hot dust. Noting that

$$
u_{\text {ext }}^{\prime}=\frac{1}{c} \int I_{\text {ext }}^{\prime} d \Omega^{\prime}=\frac{1}{c} \int I_{\text {ext }} \mathcal{D}_{\text {in }}^{-2} d \Omega \simeq u_{d i f f} \Gamma^{2}
$$

where

$$
\mathcal{D}_{\text {in }}=\frac{1}{\Gamma\left(1-\beta \cos \theta_{\text {in }}\right)}
$$

and $\theta_{i n}$ is the angle between the photon direction and the jet axis, we predict that the break in an electron energy distribution at $\gamma_{c}$ should be imprinted in the electromagnetic spectrum at frequency

$$
\nu_{E C, c} \simeq \mathcal{D}^{2} \gamma_{c}^{2} \nu_{d i f f} \simeq\left(\frac{m_{e} c^{2}}{\sigma_{T}}\right)^{2} \frac{\nu_{d i f f}}{u_{d i f f}^{2} t_{f l}^{2} \Gamma^{4}}
$$

and that the spectrum should change the slope around $\nu_{E C, c}$ by $\Delta \alpha_{X, \gamma} \simeq 0.5$. For typical energy densities expected in the BELR (broad emission line region) as well as that provided by the hot dust radiation field, the break is predicted to be located in the range of $10^{20}-10^{22} \mathrm{~Hz}$ (Sikora et al.), in agreement with observations. 35.11

In most FSRQs, during high energy flares the slope of $\gamma$-ray spectra measured by EGRET 23 is $\alpha_{\gamma} \leq 1$, while the $\mathrm{X}$-ray spectra show slopes $\alpha_{X} \simeq 0.6-0.7$. 10 The smaller than the predicted value 0.5 of $\Delta \alpha_{X, \gamma}$ can be explained by taking into account contribution to the soft and mid-X-ray bands of the SSC component 14.18 . More challenging are the so called "MeV blazars." In these objects, the spectra peak in the $300 \mathrm{keV}-30 \mathrm{MeV}$ range and the $\gamma$-ray spectra-are very soft $\left(\alpha_{\gamma}>1.4\right)$, while the X-ray spectra are very hard $\left(\alpha_{X} \leq 0.5\right) .33$ The values of $\Delta \alpha_{X, \gamma} \geq 1.0$ measured in those sources are much too large to be explained solely by the effect of cooling, and instead, it is necessary to postulate an additional break in the electron energy distribution besides $\gamma_{c}$. It is tempting to identify such a break with the break at $\gamma_{b}$, predicted by the two-step acceleration process discussed in the Section 3. External photons scattered by electrons with energies $\gamma_{b}$, are boosted to energies

$$
\nu_{E C, b} \simeq \mathcal{D}^{2} \gamma_{b}^{2} \nu_{\text {diff }} .
$$


For $2.5<\Gamma_{2} / \Gamma_{1}<10$ and $\eta_{p, t h} \sim 0.5$, the break in the electron injection function is $600<\gamma_{b}<1700$ (see Eqs. $10-12$ and Table 1), and should appear in the electromagnetic spectrum at around $1 \mathrm{GeV}$ if $\nu_{\text {diff }}=\nu_{B E L}$, and at lower energies (by a factor of $\sim 10-30$ ) if $\nu_{\text {diff }}=\nu_{I R}$. In the former case $\nu_{E C, b} \gg \nu_{E C, c}$ and the $\gamma$-ray spectra in the EGRET range should have slopes $\alpha_{\gamma} \leq 1$, while in the latter case $\nu_{E C, b} \sim \nu_{E C, c} \leq 30 \mathrm{MeV}$, and the $\gamma$-ray spectra in the EGRET range should be soft. Hence, we conclude that for blazars producing most of their radiation in the $\mathrm{GeV}$ band (and hereafter called GeV blazars) the $\gamma$-ray flux is probably produced at smaller distances (closer to the central source), where the production of $\gamma$-rays is dominated by Comptonization of broad emission lines, while the $\mathrm{MeV}$ blazar phenomenon is likely to be produced at larger distances, where production of $\gamma$-rays is dominated by the Comptonization of infrared radiation from dust.

The above scheme can also explain two other differences between $\mathrm{MeV}$ blazars and $\mathrm{GeV}$ blazars. One, already mentioned above, is that in $\mathrm{MeV}$ blazars the $\mathrm{X}$-ray spectra should be much harder than in GeV blazars. This can result from the lower contribution of the SSC component to the $\mathrm{X}$-ray band in $\mathrm{MeV}$ blazars as compared to GeV blazars (Sikora et al., in preparation). The other difference is that in the spectra of $\mathrm{MeV}$ blazars, in contrast to $\mathrm{GeV}$ blazars, the thermal UV bump is quite prominent. 33 This difference can be explained noting that magnetic fields are weaker at larger distances, and therefore in $\mathrm{MeV}$ blazars the synchrotron spectra are shifted to lower frequencies, revealing the UV bump.

\section{Magnetic fields}

Electrons with energies from the range $[\gamma ; \gamma+d \gamma]$ produce synchrotron radiation at the rate

$$
L_{\nu_{s y n}^{\prime}} d \nu_{\text {syn }}^{\prime} \sim\left(N_{\gamma} d \gamma\right)\left|\dot{\gamma}_{s y n}\right| m_{e} c^{2} \sim\left(N_{\gamma} d \gamma\right) c \sigma_{T} \gamma^{2} u_{B}^{\prime}
$$

and the angle averaged EC radiation at the rate

$$
L_{\nu_{E C}^{\prime}} d \nu_{E C}^{\prime} \sim\left(N_{\gamma} d \gamma\right)\left|\dot{\gamma}_{E C}\right| m_{e} c^{2} \sim\left(N_{\gamma} d \gamma\right) c \sigma_{T} \gamma^{2} u_{e x t}^{\prime}
$$

where

$$
\begin{gathered}
\nu_{\text {syn }}^{\prime} \sim\left(2 e / 3 \pi m_{e} c\right) \gamma^{2} B^{\prime} \\
\nu_{E C}^{\prime} \sim \gamma^{2} \nu_{e x t}^{\prime}
\end{gathered}
$$

and $u_{B}^{\prime}=B^{\prime 2} / 8 \pi$ is the magnetic energy density. Noting that $L_{\nu_{s y n}} d \nu_{s y n}=$ $L_{\nu_{s y n}^{\prime}} d \nu_{s y n}^{\prime} \mathcal{D}^{4}$ and $L_{\nu_{E C}} d \nu_{E C}=L_{\nu_{E C}^{\prime}} d \nu_{E C}^{\prime}\left(\mathcal{D}^{6} / \Gamma^{2}\right)$ we find that the ratio of 
synchrotron to EC luminosity, both produced by the same population of electrons in the same energy range, radiating in the optically thin regime, is

$$
\mathcal{R}_{s y n / E C} \equiv \frac{\nu_{s y n} L_{\nu_{s y n}}}{\nu_{E C} L_{\nu_{E C}}} \sim \frac{u_{B}^{\prime}}{u_{e x t}^{\prime}}\left(\frac{\Gamma}{\mathcal{D}}\right)^{2} .
$$

From Eqs. (17) and (21), we have

$$
u_{e x t}^{\prime} \simeq \frac{m_{e} c}{\sigma_{T}} \frac{1}{t_{f l}\left(\nu_{E C, c} / \nu_{d i f f}\right)^{1 / 2}}
$$

and inserting this into Eq. (27), we obtain

$$
u_{B}^{\prime} \simeq u_{e x t}^{\prime} \mathcal{R}_{s y n / E C}(\mathcal{D} / \Gamma)^{2} \simeq \frac{m_{e} c}{\sigma_{T}} \frac{\mathcal{R}_{\text {syn } / E C}(\mathcal{D} / \Gamma)^{2}}{t_{f l}\left(\nu_{E C, c} / \nu_{\text {diff }}\right)^{1 / 2}} .
$$

For $t_{f l} \sim 3$ day, $\mathcal{R}_{s y n / E C}=0.1, \nu_{E C, c}=10 \mathrm{MeV}$ and $\nu_{\text {diff }}=10 \mathrm{eV}$, and the observer located at $\theta_{\text {obs }}=1 / \Gamma$, Eq. (29) gives $B^{\prime} \sim \sqrt{8 \pi u_{B}^{\prime}} \sim 0.6$ Gauss.

We can estimate the energy density of the relativistic electrons and determine whether they are in equipartition with magnetic fields. Relations $E_{\text {rad }}^{\prime}=\eta_{\text {rad }} \eta_{e} E_{\text {diss }}^{\prime}$ and $E_{e}^{\prime}=\left(1-\eta_{\text {rad }}\right) \eta_{e} E_{\text {diss }}^{\prime}$ give

$$
E_{e}^{\prime}=\frac{1-\eta_{r a d}}{\eta_{\text {rad }}} E_{\text {rad }}^{\prime}
$$

where

$$
E_{\text {rad }}^{\prime} \sim L_{f l}^{\prime} t_{c o l l}^{\prime} \sim \frac{L_{f l} t_{f l}}{\mathcal{D}^{3}(\mathcal{D} / \Gamma)^{2}} .
$$

Hence, energy density of the relativistic electrons is

$$
u_{e}^{\prime}=\frac{E_{e}^{\prime}}{\pi a^{2} \Delta_{s h}^{\prime}} \simeq \frac{1-\eta_{\text {rad }}}{\eta_{\text {rad }}} \frac{L_{f l}}{2 \pi c^{3}\left(a / a_{\Gamma}\right)^{2} t_{f l}^{2} g \mathcal{D}^{6}(\mathcal{D} / \Gamma)^{2}},
$$

where $a \leq a_{\Gamma}=c t_{f l} \mathcal{D}$ is the cross-sectional dimension (radius) of a shocked plasma sheet, and $\Delta_{s h}^{\prime}=2 c t_{f l} \mathcal{D} g$ is the thickness of the sheet. The scaling factor $g$, which relates the thickness of the shocked plasma sheet to the time scale of the flare/collision, depends on $\Gamma_{2} / \Gamma_{1}$ and on the adiabatic index. The analytical form of $g$ is derived and presented in Moderski et al. (in preparation), and the example values of $g$ are shown in the Table 1.

Combining Eqs. (29) and (32), we obtain

$$
\frac{u_{B}^{\prime}}{u_{e}^{\prime}} \simeq \frac{2 \pi m_{e} c^{4}}{\sigma_{T}} \frac{\eta_{\text {rad }}}{1-\eta_{\text {rad }}} \frac{g\left(a / a_{\Gamma}\right)^{2} \mathcal{R}_{\text {syn } / E C}}{\left(\nu_{E C, c} / \nu_{\text {diff }}\right)^{1 / 2}} \frac{t_{f l}}{L_{f l}} \mathcal{D}^{6}(\mathcal{D} / \Gamma)^{4} .
$$


Table 1: Dependence of the internal shock parameters on $\Gamma_{2} / \Gamma_{1}$. Values of $g_{0}$ and $g$ are calculated for the adiabatic index of the shocked plasma $\hat{\gamma}=5 / 3$. Analytical formulas for $g_{0}$ and $g$ are presented in Moderski et al. (in preparation).

\begin{tabular}{|c|c|c|c|c|c|c|c|c|}
\hline$\Gamma_{2} / \Gamma_{1}$ & $\eta_{\text {diss }}$ & $\kappa$ & $\gamma_{p, t h}-1$ & $\gamma_{b}$ & $\bar{\gamma}_{i n j}$ & $n_{+} / n_{p}$ & $g_{0}$ & $g$ \\
\hline 1.5 & 0.020 & 0.020 & 0.010 & 265 & 15.3 & 0.2 & 0.273 & 0.067 \\
2.5 & 0.097 & 0.107 & 0.053 & 609 & 18.0 & 2.4 & 0.641 & 0.150 \\
5.0 & 0.255 & 0.342 & 0.171 & 1120 & 20.4 & 7.6 & 1.237 & 0.255 \\
10.0 & 0.425 & 0.739 & 0.370 & 1722 & 22.2 & 15.5 & 2.025 & 0.346 \\
\hline
\end{tabular}

The equation demonstrates very strong dependence of $u_{B}^{\prime} / u_{e}^{\prime}$ on $\Gamma$ and on the observed angle $\theta_{\text {obs }}$. Provided that blazars are observed at $\theta_{o b s} \sim 1 / \Gamma$, the equipartition condition, $u_{B}^{\prime} \sim u_{e}^{\prime}$, implies

$$
\Gamma \simeq\left(\frac{\sigma_{T}}{2 \pi m_{e} c^{4}} \frac{\left(\nu_{E C, c} / \nu_{d i f f}\right)^{1 / 2}}{\mathcal{R}_{\text {syn } / E C} g\left(a / a_{\Gamma}\right)^{2}} \frac{1-\eta_{\text {rad }}}{\eta_{\text {rad }}} \frac{L_{f l}}{t_{f l}}\right)^{1 / 6}
$$

For flares with luminosities $L_{f l} \sim 10^{48-49} \mathrm{ergs} \mathrm{s}^{-1}$ and time scales $t_{f l} \sim 3 \mathrm{~d}$, and assuming $a=a_{\Gamma}, \Gamma_{2} / \Gamma_{1}=2.5$, and $\eta_{r a d}=0.5$, we obtain $\Gamma \sim 18-26$.

\section{Pair content}

The pair content is

$$
\frac{n_{+}}{n_{p}}=\left(\frac{n_{e}}{n_{p}}-1\right) / 2
$$

where

$$
\begin{gathered}
\frac{n_{e}}{n_{p}}=\eta_{e} \frac{m_{p} \kappa}{m_{e}\left(\bar{\gamma}_{i n j}-1\right)} \\
\bar{\gamma}_{i n j}=\frac{\int_{1}^{\gamma_{\max }} \gamma Q d \gamma}{\int_{1}^{\gamma_{\max }} Q d \gamma}
\end{gathered}
$$

and $Q$ is the electron injection function. For a double power-law injection function, $Q \propto \gamma^{-p_{L}}$ for $1<\gamma<\gamma_{b}$, and $Q \propto \gamma^{-p_{H}}$ for $\gamma_{b}<\gamma<\gamma_{\max }$, and observationally suggested indices, $1<p_{L}<2$ and $p_{H}>2$,

$$
\bar{\gamma}_{i n j} \simeq \frac{\left(p_{L}-1\right)\left(p_{H}-p_{L}\right)}{\left(2-p_{L}\right)\left(p_{H}-2\right)} \gamma_{b}^{2-p_{L}} .
$$

For $p_{L}=1.8\left(\rightarrow \alpha_{X}=\left(p_{L}-1\right) / 2 \sim 0.45\right.$ and $\alpha_{\gamma}=p_{L} / 2 \sim 0.9$ in the EGRET band of GeV blazars $)$, and $p_{H}=2.8\left(\rightarrow \alpha_{\gamma}=p_{H} / 2=1.4\right.$ in the EGRET band of MeV blazars), and $2.5<\Gamma_{2} / \Gamma_{1}<10$, the number of pairs per proton is $2.4<n_{+} / n_{p}<15.5$ (see Table 1 ). 


\section{The power of a jet}

Energy carried by the cold inhomogeneities prior to the collision is (see Eq. 6)

$$
E_{i} \simeq \frac{N_{p}}{2} m_{p} c^{2} \Gamma_{i} \simeq \frac{L_{f l} t_{f l}}{\kappa f \mathcal{D}^{3} \eta_{\text {rad }} \eta_{e}} \Gamma_{i}
$$

where $i=1,2$. The time it takes to eject the inhomogeneities from the central source is

$$
t_{e j, i} \simeq \frac{\lambda_{0}}{c \beta_{i} \Gamma_{i}} \simeq \frac{\mathcal{D}}{\Gamma_{i}} g_{0} t_{f l}
$$

where $\lambda_{0}=g_{0} c \mathcal{D} t_{f l}$ is the proper length of an inhomogeneity (the thickness of a shell segment), and $g_{0}$ is the factor depending on $\Gamma_{2} / \Gamma_{1}$ and on the adiabatic index of the shocked plasma (see Table 1). The time-averaged power of a jet is

$$
P_{j} \sim \Psi \frac{E_{1}+E_{2}}{t_{e j, 1}+t_{e j, 2}} \simeq \Psi \frac{N_{p} m_{p} c^{3} \Gamma^{2}}{\lambda_{0}} \simeq \Psi \frac{L_{f l}}{2 \eta_{e} \eta_{r a d} \kappa g_{0}} \frac{\Gamma^{2}}{f \mathcal{D}^{4}},
$$

where $\Psi$ is the duty cycle factor. For $L_{f l} \sim 10^{48} \mathrm{erg} \mathrm{s}^{-1}, \mathcal{D} \sim \Gamma \sim 15$, $\Gamma_{2} / \Gamma_{1} \sim 2.5, \eta_{e} \sim \eta_{\text {rad }} \sim 0.5$ and $\Psi \sim 0.1$, Eq. (41) gives $P_{j} \sim 3 \times 10^{46} \mathrm{erg} \mathrm{s}^{-1}$.

\section{$8 \quad \mathrm{X}$-ray precursors}

Prior to the collision, electrons in inhomogeneities are cold, but being pulled by streaming protons theyscatter external photons and produce the beamed bulk-Compton radiation. 229 The spectrum of this radiation peaks around

$$
\nu_{i} \simeq \mathcal{D}_{i} \Gamma_{i} \nu_{d i f f}
$$

and has luminosity

$$
L_{X, i} \simeq \delta N_{e, i}\left|\dot{\epsilon}_{e, i}\right| \mathcal{D}_{i}^{4}
$$

where

$$
\begin{gathered}
\delta N_{e, i}=\left(N_{e} / 2\right) \operatorname{Min}\left[1 ; r_{B C} / \lambda_{0} \mathcal{D}_{i}\right], \\
N_{e} \simeq \frac{E_{e, i n j}^{\prime}}{\bar{\gamma}_{i n j} m_{e} c^{2}} \simeq \frac{\eta_{e} E_{d i s s}^{\prime}}{\bar{\gamma}_{i n j} m_{e} c^{2}} \simeq \frac{L_{f l} t_{f l}}{f \mathcal{D}^{3} \eta_{\text {rad }} \bar{\gamma}_{i n j} m_{e} c^{2}}, \\
\left|\dot{\epsilon}_{e, i}\right| \simeq \Gamma_{i}^{2} c \sigma_{T} u_{e x t}, \\
\mathcal{D}_{i} \equiv \frac{1}{\Gamma_{i}\left(1-\beta_{i} \cos \theta_{o b s}\right)},
\end{gathered}
$$

$r_{B C}$ is the distance within which most of the bulk Compton radiation is produced. The factor $\operatorname{Min}\left[1 ; r_{B C} / \lambda_{0} \mathcal{D}_{i}\right]$ in Eq. (44) expresses the fact that in 
general, due to light travel effects and the finite length of the source, not all electrons contribute to the radiation observed at a given instant.

Bulk-Compton flares from cold inhomogeneities are expected to precede the nonthermal flares by

$$
\delta t_{i} \sim \frac{r_{f l}}{c \Gamma_{i} \mathcal{D}_{i}} \simeq \frac{\mathcal{D} \Gamma}{\mathcal{D}_{i} \Gamma_{i}} t_{f l} \frac{\Delta r_{\text {coll }}}{r_{f l}} .
$$

Above formulae are very approximate, because they do not take into account that the observed bulk Compton radiation at any given instant includes contributions from a finite distance range over which $u_{e x t}$ can change significantly. However, making the conservative assumption that the jet is developed (accelerated and collimated) not earlier than at a distance where electron energy losses are already dominated by Compton interactions with the broad emission line flux rather than the direct radiation from an accretion disc, we can calculate the minimum bulk Compton luminosity, using an approximation that $u_{e x t} \simeq u_{B E L} \sim$ const for $r<r_{B E L}$ and 0 for $r>r_{B E L}$. Noting that $r_{B E L} \sim 3 \times 10^{17} \sqrt{L_{U V, 46}} \mathrm{~cm}$ and $u_{B E L} \sim 0.03 \mathrm{erg} \mathrm{cm}^{-3}$ (Peterson 2 ), we find that for $\theta_{o b s}=1 / \Gamma=1 / 15, \Gamma_{2} / \Gamma_{1}=2.5, L_{f l}=10^{48} \mathrm{erg} \mathrm{s}^{-1}$ and $t_{f l}=3$ day, the precursor produced by the faster-moving inhomogeneity should have luminosity $L_{X, 2} \sim 3 \times 10^{46} \mathrm{ergs} \mathrm{s}^{-1}$; should peak at around $\mathrm{h} \nu_{2} \simeq 3.2 \mathrm{keV}$; and should precede the nonthermal flare by $\delta t_{2} \simeq 0.7 t_{f l}$. The precursor produced by the slower moving inhomogeneity would have luminosity $L_{X, 1} \sim 5 \times 10^{45}$ $\mathrm{erg} \mathrm{s}^{-1}$; would peak around $\mathrm{h} \nu_{1} \simeq 1.8 \mathrm{keV}$; and would precede the nonthermal flare by $\delta t_{1} \simeq 1.8 t_{f l}$. Since typical soft $\mathrm{X}$-ray luminosities are of the order $10^{46} \mathrm{erg} \mathrm{s}^{-1}$, we can infer from the above estimates that jets propagating through much denser quasi-isotropic radiation fields than would be provided by the BELR are predicted to produce much stronger soft/mid X-ray radiation than is observed. This puts severe constrains on the jet structure at $10^{2}-10^{3}$ gravitational radii from the $\mathrm{BH}$, where quasi-isotropic radiation is strongly dominated by the very dense direct radiation of an accretion disc and its corona. Hence, the lack of very large soft $\mathrm{X}$-ray excesses suggests that jets near their bases are very wide (meaning that they have relatively large opening angle) and/or are slower than at larger distances.

\section{Summary}

Multi-wavelength observations of blazars provide a valuable tool allowing us to learn about the structure and physical properties of sub-parsec-scale jets in quasars. The observations suggest:

- Rapid flares and transverse magnetic fields inferred from polarization data $\rightarrow$ Streams of matter in small-scale jets are unsteady, and collisions between 
inhomogeneities propagating down the jet with different radial velocities can easily form transverse shocks;

- Large powers of jets and relatively low soft/mid X-ray luminosities $\rightarrow$ Inertia of jets is dominated by protons and the number of pairs per proton is $<15$;

- Very hard X-ray spectra $\rightarrow X$-rays and $\gamma$-rays are produced by electrons accelerated directly, just as is the case for the synchrotron radiation, rather than being injected following the proton-induced pair cascades;

- Differences between properties of $\mathrm{GeV}$ and $\mathrm{MeV}$ blazars $\rightarrow E C$ model can explain these differences assuming a two-power-law electron injection function. The break in the injection function can be related to the threshold energy for the Fermi acceleration of electrons;

- Luminous, short term $\gamma$-ray flares in FSRQs $\rightarrow$ magnetic fields are much below equipartition with the relativistic plasma, unless $\Gamma \geq 18-26$;

- No prominent spectral signatures of bulk Compton process in the soft/mid $\mathrm{X}$-ray bands $\rightarrow$ Collimation and/or acceleration of jets occurs beyond $10^{2}-10^{3}$ gravitational radii from the central source.

\section{Acknowledgments}

This work was partially supported by Polish KBN grant 5 P03D 00221 and NASA Chandra observing grant to Stanford University. M.S. thanks the Stanford Linear Accelerator Center for its hospitality during the completion of this work, and the Korean Institute for Advanced Study for hosting this interesting meeting.

\section{References}

1. Begelman, M.C., \& Cioffi, D.C. 1989, ApJ, 345, L21.

2. Begelman, M.C., \& Sikora, M. 1987, ApJ, 322, 650.

3. Błażejowski, M., Sikora, M., Moderski, R., \& Madejski, G.M. 2000, ApJ, $545,107$.

4. Celotti, A., \& Fabian, A.C. 1993, MNRAS, 228.

5. Celotti, A., Ghisellini, G., \& Chiaberge, M. 2001, MNRAS, 321, L1.

6. Comastri, A., Fossati, G., Ghisellini, G., \& Molendi, S. 1997, ApJ, 480, 534.

7. Dermer, C.D. 1995, ApJ, 446, L63.

8. Dermer, C.D., \& Chiang, J. 1998, NewA, 3, 157.

9. Dieckmann, M.E., McClements, K.G., Chapman, S.C., Dendy, R.O., \& Drury, L.O'C. 2000, A\&A, 356, 377.

10. Donato, D., Ghisellini, G., Tagliaferri, G., \& Fossati, G. 2001, A\&A, 375, 739. 
11. Fossati, G., Maraschi, L., Celotti, A., Comastri, A., \& Ghisellini, G. 1998, MNRAS, 299, 433.

12. Homan, D.C., Attridge, J.M., \& Wardle, J.F.C. 2001, 556, 113.

13. Impey, C.D., Lawrence, C.R., \& Tapia, S. 1991, ApJ, 375, 46.

14. Inoue, S., \& Takahara, F. 1996, ApJ, 463, 555.

15. Jones, F.C. 1994, ApJS, 90, 561.

16. Jorstad, S.G., Marscher, A., Mattox, J.R., Wehrle, A.E., Bloom, S.D., \& Yurchenko, A.V. 2001, ApJS, 134, 181.

17. Komissarov, S.S., \& Falle, S.A.E.G. 1997, MNRAS, 288, 833.

18. Kubo, H., et al. 1998, ApJ, 504, 693.

19. Lister, M.L. 2001, ApJ, in press astro-ph/0107594.

20. Madejski, G.M., Sikora, M., Jaffe, T., Błażejowski, M., Jahoda, K., \& Moderski, R. 1999, ApJ, 521, 145.

21. Mannheim, K., \& Biermann, P.L. 1992, A\&A, 253,, L21.

22. Peterson, B.M. 1993, PASP, 105, 247.

23. Pohl, M., Hartman, R.C., Jones, B.B., \& Sreekumar, P. 1997, A\&A, 326, 51.

24. Rawlings, S., \& Saunders, R. 1991, Nature, 349, 138.

25. Romanova, M.M., \& Lovelace, R.V.E. 1992, A\&A, 262, 26.

26. Sanders, R.H. 1983, ApJ, 266, 73.

27. Shimada, N., \& Hoshino, M. 2000, ApJ, 543, L67.

28. Sikora, M., Begelman, M.C., \& Rees, M.J. 1994, ApJ, 421, 153.

29. Sikora, M., \& Madejski, G.M. 2000, ApJ, 534, 109.

30. Sikora, M., \& Madejski, G.M. 2001, AIP Conference Proceedings, 558, 275.

31. Spada, M., Ghisellini, G., Lazzati, D., Celotti, A. 2001, MNRAS, 325, 1559.

32. Stevens, J.A., Robson, E.I., \& Holland, W.S. 1996, ApJ, 462, L23.

33. Tavecchio, F., Maraschi, L., Sambruna, R.M., \& Urry, C.M. 2000, ApJ, $544, \mathrm{~L} 23$.

34. Vermeulen, R.C., \& Cohen, M.H. 1994, ApJ, 430, 467.

35. von Montigny, C., et al. 1995, ApJ, 440, 525.

36. Wardle, J.F.C., \& Aaron, S.E. 1997, MNRAS, 286, 425.

37. Zensus, J.A. 1997, ARAA, 35, 607. 